\title{
Exhaled nitric oxide as a marker of asthma control in smoking patients
}

\author{
A. Michils*, R. Louis", R. Peché ", S. Baldassarre* and A. Van Muylem*
}

\begin{abstract}
Exhaled nitric oxide fraction ( $\mathrm{FeNO}$ ), which is a reliable marker of eosinophilic airway inflammation, is partially suppressed by tobacco smoking. Consequently, its potential as a biomarker in asthma management has never been evaluated in smoking patients. In the present study, the authors tested the validity of $F$ eNO to predict asthma control in this population.

FeNO and the Asthma Control Questionnaire (ACQ) were recorded at least once in 411 nonsmoking (345 with at least two visits) and 59 smoking (51 with at least two visits) asthma patients.
\end{abstract}

Despite similar mean ACQ scores (1.5 versus 1.7), FeNO was reduced in smoking asthmatics (18.1 ppb versus $33.7 \mathrm{ppb}$ ). A decrease in $\mathrm{FeNO}$ of $<20 \%$ precludes asthma control improvement in nonsmoking (negative predictive value (NPV) $78 \%$ ) and in smoking patients (NPV $72 \%$ ). An increase in $\mathrm{FeNO}<\mathbf{3 0} \%$ is unlikely to be associated with deterioration in asthma control in both groups of patients (NPV $=86 \%$ and $84 \%$ in nonsmoking and smoking patients, respectively).

It is concluded that, even in smokers, sequential changes in FeNO have a relationship with asthma control. The present study is the first to indicate that cigarette smoking does not obviate the clinical value of measuring $F$ eNO in asthma among smokers.

\section{KEYWORDS: Asthma control, exhaled nitric oxide, tobacco smoking}

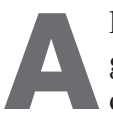

lthough the debate is not over, it is generally accepted that the exhaled nitric oxide fraction $(\mathrm{FeNO})$ has the potential to be useful in the management of asthma [1-6]. However, several factors confounding FeNO measurement have been recognised [7]. Among them, tobacco smoking has been consistently shown to reduce FeNO levels [6, 8-15], by a factor varying from 0.63 to 0.80 according to the multivariate analyses that have compared FeNO in smokers and nonsmokers $[6,14,15]$. The mechanism by which smoking causes $\mathrm{FeNO}$ reduction is not fully understood, but may include reduction in nitric oxide (NO) synthesis due to feedback inhibition induced by high concentrations of $\mathrm{NO}$ contained in cigarette smoke [9]. NO oxidation or interaction with other molecules present in tobacco smoke might also occur [16]. However, regardless of the mechanism of FeNO reduction reported in smokers, it is generally assumed that FeNO should not be assessed in asthmatic patients who smoke. Perhaps, consequently, this population $(\sim 25 \%$ of adult asthma patients [17]) has been excluded from clinical trials that have explored the potential of $\mathrm{FeNO}$ as a biomarker in asthma management. Even in the present authors' own studies, where it has recently been shown that FeNO is a reliable marker of asthma control over time in unselected patients, smoking patients were not enrolled [18]. Interestingly, the present authors' previous study [18] strongly suggested that it is the change in FeNO values, rather than absolute cut-off points (i.e. individualised FeNO profiles), that may be meaningful for the longitudinal assessment of asthma control in daily practise. Therefore, in the present study, the authors investigated whether, despite the FeNO reduction reported in smoking asthma patients [6, 8-15], changes in FeNO might also be significantly related to changes in asthma control in this population.

To do this, FeNO was monitored on several occasions in smoking and nonsmoking patients attending a tertiary asthma clinic. Its ability to reflect improvement or worsening of asthma control over time was compared in both groups, using the Asthma Control Questionnaire (ACQ) as a gold standard for the assessment of asthma control [19].

\section{METHODS}

\section{Subjects}

Between January 1, 2004, and July 30, 2008, 411 adult nonsmokers and 59 adult smokers
AFFILIATIONS

Chest Depts, ${ }^{*}$ CUB Erasme, Brussels,

${ }^{\#} \mathrm{CHU}$ Sart-Tilmant, Liège, and 'CHU André Vésale, Montigny-leTilleul, Belgium.

\section{CORRESPONDENCE}

A. Van Muylem

Chest Dept

CUB Erasme

808 Route de Lennik

1070 Brussels

Belgium

Fax: 3225554411

E-mail: avmuylem@ulb.ac.be

Received:

October 132008

Accepted after revision:

January 032009

\section{SUPPORT STATEMENT}

AstraZeneca provided a grant for the exhaled biomarker laboratory.

\section{STATEMENT OF INTEREST}

A statement of interest for this study can be found at

www.erj.ersjournals.com/misc/ statements.dtl 
attending the Allergy and Asthma clinic in the Chest Dept of the Erasme University Hospital, Brussels, Belgium, for treatment of persistent asthma diagnosed according to standard criteria [20] were enrolled in the study.

The present study was approved by the local ethics committee, and patients signed to give informed consent.

\section{Study procedures and design}

\section{Study design}

The present study is a post hoc analysis of an existing database that is continuously updated. A significant part of the database was reported in the present authors' previously published study, which documented a relationship between asthma control and FeNO in nonsmoking asthma patients [18]. The present analysis focuses on whether current smoking annuls the validity of FeNO measurements to predict asthma control. ACQ scores and FeNO were recorded independently on one or more occasions for each patient, including smokers who were excluded from the initial analysis for reasons mentioned earlier. At each visit, asthma treatment was adjusted according to the Global Initiative for Asthma (GINA) guidelines recommendations [20], regardless of ACQ score or FeNO value, which were recorded separately.

Since optimal asthma control appears more difficult to achieve in smoking patients [21], the 1.5 optimum cut-off point identifying poorly controlled asthma [22] was selected as the reference ACQ score in the receiver operating characteristic (ROC) curve analysis. For clarity's sake, it was considered that an ACQ score of $<1.5$ identified asthma that is controlled (i.e. partly or well controlled), whereas an ACQ score of $\geqslant 1.5$ identified uncontrolled asthma. Using the ROC curve analysis, the present authors assessed the ability of FeNO to: 1) reflect asthma control cross-sectionally using an ACQ threshold of 1.5 ; 2) detect a significant improvement or worsening of asthma control that resulted in a change from uncontrolled $(\mathrm{ACQ} \geqslant 1.5)$ to controlled (ACQ <1.5) asthma, either respectively or vice versa; and 3) detect a significant improvement or worsening of asthma control defined as a decrease or increase in ACQ of $\geqslant 0.5$, even though it was not large enough to result in a change of the asthma control status.

Patients treated with low $(\leqslant 500 \mu \mathrm{g}$ beclomethasone diproprionate (BDP) eq $\cdot$ day $^{-1}$ ) and high-to-moderate $(>500 \mu \mathrm{g} \mathrm{BDP}$ eq $\cdot$ day $^{-1}$ ) inhaled corticosteroid (ICS) doses were considered separately. Indeed, in the present authors' previous study [18], it was found that the overall ability of FeNO to reflect asthma control was reduced in patients using high ICS doses.

\section{Study procedures}

Asthma Control Questionnaire

Asthma control was assessed using a French translation of the short version of the ACQ of JUNIPER et al. [23]. This version does not include FEV1 rating. Patients subjectively evaluate the degree of impairment caused by their asthma during the preceding 7 days by responding to six questions using a sevenpoint scale: a score of 0 indicates no impairment and a score of 6 indicates maximal impairment. The total ACQ score is the mean of the six responses, thus varying between 0 (totally controlled asthma) and 6 (totally uncontrolled asthma). A score of $>1.5$ is used to identify poorly controlled asthma [22]. A change of 0.5 in the ACQ score is considered to be the minimum change that is clinically relevant [22].

\section{FeNO}

FeNO was measured before any forced expiratory manoeuvres using a daily calibrated LR 2000 chemoluminescence analyser (Logan Research Ltd, Rochester, UK) with online measurement of a single exhalation at a flow rate of $50 \mathrm{~mL} \cdot \mathrm{s}^{-1}$ (American Thoracic Society (ATS)/European Rspiratory Society (ERS) standard) [24]. FeNO levels were read at the plateau corresponding to $70-80 \%$ of the carbon dioxide curve. Absolute FeNO values are expressed in $\mathrm{ppb}$, and changes in $\mathrm{FeNO}$ are expressed as a percentage of the initial value $(\Delta \%)$.

\section{Statistical methods}

ROC curve analysis was performed in the whole population, as well as in the two different subgroups, i.e. patients treated by low and high-to-moderate doses. The area under the ROC curve (AUC) was computed, and its difference from 0.5 was statistically evaluated. For a given type of assessment, the optimal cut-off value was determined for the whole population by maximising the Youden's index [25], i.e. the true-positive rate (sensitivity) minus the false-positive rate (1-specificity; see online supplementary material). Geometrically, this index is the vertical distance between the ROC curve and the first bisector. The cut-off value corresponding to the maximum value of Youden's index was then used to derive sensitivity (Se), specificity (Sp), positive predictive values (PPV) and negative predictive values (NPV), and accuracy in the whole population and in the subgroups of patients. In the online supplementary material, Se, Sp, PPV, NPV and accuracy may be found for other cut-off values, as well as the amounts of true-positive, true-negative, false-positive and false-negative cases (contingency tables).

Unpaired t-tests were used when considering forced expiratory volume in $1 \mathrm{~s}$ (FEV1) and log-transformed FeNO values, and Mann-Whitney U-tests when considering ICS doses and ACQ scores. Proportions were compared using a Chi-squared test. The limit of significance was a p-value of 0.05 .

\section{RESULTS}

Of the 411 nonsmoking patients and 59 smoking patients, 345 and 51, respectively, were seen at least twice, representing 646 and 92 pairs of successive visits for nonsmoking and smoking patients, respectively (the median time between two visits for nonsmoking patients was 88 days, range 10-1,255 days, interquartile interval 42-189; and for smoking patients it was 93 days, range 7-525 days, interquartile interval 49-182). Table 1 presents demographic data for the nonsmoking and smoking groups, as well as FeNO, FEV1, ACQ score and ICS dose values at study onset for the total studied population (included in the cross-sectional analysis) and for the subgroup of patients who were seen at least twice (included in the longitudinal analysis).

Tables 2-5 display the cut-off values (resulting from Youden's index maximisation), the number of positive and total cases, and therefore the prevalence, the Se, the Sp, the PPV and NPV, the accuracy and the p-value allowing rejection (or not) of the null hypothesis $\mathrm{AUC}=0.5$. 
TABLE 1 Demographic data and indices values at study onset

\begin{tabular}{|c|c|c|c|c|c|c|}
\hline & \multicolumn{3}{|c|}{ Total } & \multicolumn{3}{|c|}{ Patients seen at least twice } \\
\hline & Nonsmokers & Smokers & p-value ${ }^{\#}$ & Nonsmokers & Smokers & p-value ${ }^{\#}$ \\
\hline Subjects $n$ & 411 & 59 & & 345 & 51 & \\
\hline Age yrs & $41 \pm 16$ & $38 \pm 11$ & 0.39 & $41 \pm 16$ & $39 \pm 11$ & 0.47 \\
\hline Male/female $n$ & $195 / 216$ & $34 / 25$ & 0.14 & 219/126 & $26 / 25$ & 0.08 \\
\hline ICS dose $e^{\varphi,+}$ & $250(0-2000)$ & $500(0-2000)$ & 0.50 & $250(0-2000)$ & $500(0-2000)$ & 0.37 \\
\hline FeNO $\mathrm{ppb}^{\S}$ & $33.7(14.3-79.2)$ & $18.1(6.9-47.5)$ & $<0.001$ & $34.8(14.6-83.0)$ & $18.5(6.1-55.5)$ & $<0.001$ \\
\hline FEV1 \% pred & $85.6 \pm 15.7$ & $86.2 \pm 17.9$ & 0.80 & $85.0 \pm 20.6$ & $86.5 \pm 18.0$ & 0.79 \\
\hline
\end{tabular}

Data are presented as mean $\pm \mathrm{SD}$, median (range) $\left(^{\natural}\right)$, or geometrical mean (geometrical interval) $\left({ }^{\S}\right)$, unless otherwise indicated. ACQ: Asthma Control Questionnaire ICS: inhaled corticosteroid; FeNO: exhaled nitric oxide fraction; FEV1: forced expiratory volume in $1 \mathrm{~s}$; \% pred: \% predicted. \#: comparison between nonsmoking and smoking group; ${ }^{+}$: ICS dose in $\mu$ g equivalents beclomethasone diproprionate per day ${ }^{-1}$ with the exception of $F$ eNO, nonsmoking and smoking groups were statistically similar.

\section{Cross-sectional assessment of asthma control}

Asthma control was cross-sectionally assessed at study onset for 411 nonsmoking and 59 smoking asthma patients. Controlled asthma (ACQ score of $<1.5$ ) was considered as a positive event. Table 2 shows that, in smoking asthma patients, FeNO is unable to cross-sectionally assess asthma control.

\section{Assessment of change in asthma control between pairs of visits}

Change from uncontrolled $(A C Q$ score $\geqslant 1.5)$ to controlled $(A C Q$ score <1.5) asthma

In nonsmoking and smoking patients, asthma was uncontrolled at visit 1 in 283 pairs (out of 646) and 52 pairs (out of

\section{TABLE 2 Cross-sectional assessment of asthma control}

\begin{tabular}{lcc} 
& Nonsmokers & Smokers \\
\hline Total events n & 411 & 59 \\
Positive cases n & 197 & 15 \\
Prevalence & 48 & 25 \\
Cut-off ppb & 50 & 25 \\
Sensitivity & 72 & 66 \\
Specificity & 56 & 48 \\
PPV & 61 & 30 \\
NPV & 68 & 81 \\
Accuracy & 64 & 53 \\
p-value & $<0.001$ & 0.39 \\
\hline
\end{tabular}

Data are presented as \%, unless otherwise indicated. PPV: positive predictive value; NPV: negative predictive value. ${ }^{\#}$ : the $\mathrm{p}$-value was defined as the statistical significance of rejecting an area under the curve of 0.5. A positive event was defined as controlled asthma (Asthma Control Questionnaire score $<1.5)$. A true-positive case was defined as an exhaled nitric oxide fraction ( $F \mathrm{eNO}$ ) of less than or the same as the cut-off value associated with a controlled asthma. FeNO does not discriminate for or against cross-sectionally controlled versus uncontrolled asthma in smoking patients $(p=0.39)$.
92), respectively. A change to controlled asthma (spontaneous as well as treatment-induced) at visit 2 was considered as a positive event. In nonsmoking and smoking patients, this was observed on 133 and 17 occasions, respectively.

Table 3 shows that FeNO exhibits high operating characteristics in both nonsmoking and smoking groups. The cut-off values for decreases in FeNO which had the highest NPVs for establishing control were $30 \%$ in nonsmokers and $20 \%$ in smokers.

\begin{tabular}{|c|c|c|}
\hline \multirow[t]{2}{*}{ TABLE 3} & \multicolumn{2}{|c|}{$\begin{array}{l}\text { Assessment of a change from uncontrolled } \\
\text { (Asthma Control Questionnaire }(A C Q) \text { score of } \\
\geqslant 1.5) \text { to controlled (ACQ score }<1.5 \text { ) asthma }\end{array}$} \\
\hline & Nonsmokers & Smokers \\
\hline Total events $\mathbf{n}$ & 283 & 52 \\
\hline Positive cases $n$ & 133 & 17 \\
\hline Prevalence & 47 & 33 \\
\hline Cut-off & -30 & -20 \\
\hline Sensitivity & 68 & 71 \\
\hline Specificity & 71 & 66 \\
\hline PPV & 68 & 50 \\
\hline NPV & 72 & 82 \\
\hline Accuracy & 70 & 67 \\
\hline p-value ${ }^{\#}$ & $<0.001$ & 0.016 \\
\hline
\end{tabular}

Data are presented as \%, unless otherwise indicated. PPV: positive predictive value; NPV: negative predictive value. ${ }^{\#}$ : the $p$-value was defined as the statistical significance of rejecting an area under the curve of 0.5 . A positive event was defined as a change from uncontrolled ( $A C Q$ score of $\geqslant 1.5$ ) to controlled (ACQ score <1.5) asthma. A true-positive case was defined as exhaled nitric oxide fraction ( $F_{\mathrm{eNO}}$ ) change of less than or the same as the cutoff value (e.g. $-40 \%)$ associated with a positive event. FeNO exhibited similar operating characteristics in both the nonsmoking and smoking group. In particular, a high NPV was observed. 


\begin{tabular}{lcc}
\hline TABLE 4 & $\begin{array}{l}\text { Assessment of a change from controlled } \\
\text { (Asthma Control Questionnaire (ACQ) score } \\
<1.5) \text { to uncontrolled (ACQ score } \geqslant 1.5) \text { asthma }\end{array}$ \\
& Nonsmokers & Smokers \\
\hline Total events $\mathbf{n}$ & 360 & \\
Positive cases $\mathbf{n}$ & 65 & 40 \\
Prevalence & 18 & 10 \\
Cut-off & +50 & 25 \\
Sensitivity & 42 & +50 \\
Specificity & 75 & 68 \\
PPV & 26 & 87 \\
NPV & 86 & 63 \\
Accuracy & 69 & 89 \\
p-value & 0.001 & 83 \\
\hline
\end{tabular}

Data are presented as \%, unless otherwise indicated. PPV: positive predictive value; NPV: negative predictive value. ${ }^{\#}$ : the $p$-value was defined as the statistical significance of rejecting an area under the curve of 0.5 . A positive event was defined as a change from controlled (ACQ score <1.5) to uncontrolled (ACQ score $\geqslant 1.5$ ) asthma. A true-positive case was defined as an exhaled nitric oxide fraction ( $\mathrm{FeNO}$ ) change of more than or the same as the cut-off value associated with a positive event. FeNO exhibited similar operating characteristics in both the nonsmoking and smoking group. In particular, a high NPV was observed.

Change from controlled (ACQ score $<1.5$ ) to uncontrolled (ACQ score $\geqslant 1.5$ ) asthma

In nonsmoking and smoking patients, asthma was controlled at visit 1 in 360 pairs (out of 643) and 40 pairs (out of 92), respectively. A change to uncontrolled asthma at visit 2 was considered as a positive event. In nonsmoking and smoking patients, this was observed on 65 and 10 occasions, respectively.
Table 4 shows that $F$ eNO exhibits high operating characteristics in both nonsmoking and smoking groups. The cut-off values for an increase in FeNO, which had the highest NPVs for a change to uncontrolled asthma was $50 \%$ in both nonsmokers and smokers.

Improvement of asthma control $(\triangle \mathrm{ACQ}<-0.5)$

A significant improvement in asthma control between two consecutive visits was considered to be a positive event. As a whole, in nonsmoking and smoking patients, this occurred on 257 and 40 occasions, respectively.

Table 5 shows that, in the entire population, FeNO exhibited similar operating characteristics in nonsmoking and smoking patients. Figure 1 illustrates this feature.

When considering the subgroup of smoking patients treated with $>500 \mu \mathrm{g}$ eqivalents $\mathrm{BDP} \cdot \mathrm{day}^{-1}$, FeNO was no longer significant in assessing an improvement of asthma control.

Worsening of asthma control $(\triangle \mathrm{ACQ}>0.5)$

A significant worsening of asthma control between two consecutive visits was considered a positive event. As a whole, in nonsmoking and smoking patients, this occurred on 161 and 26 occasions, respectively.

Table 6 shows that, as for improvement assessment, FeNO exhibited analogous operating characteristics in nonsmoking and smoking patients. With a cut-off value at $30 \%$ change, a high NPV was observed in both groups. When considering the subgroup of smoking patients treated with $>500 \mu \mathrm{g}$ eqivalents $\mathrm{BDP} \cdot \mathrm{day}^{-1}, \mathrm{FeNO}$ operating characteristics in assessing asthma control worsening are less significant.

In both improvement (table 5) and worsening (table 6) assessment of asthma control, the present authors considered a subgroup of pairs of visits with an initial ACQ score of $<2$ as well as a subgroup of pairs of visits without ICS dose

TABLE 5 Assessment of asthma control improvement (change in Asthma Control Questionnaire $(\triangle A C Q)<-0.5)$

\begin{tabular}{|c|c|c|c|c|c|c|c|c|c|c|}
\hline & \multicolumn{5}{|c|}{ Nonsmokers ${ }^{\#}$} & \multicolumn{5}{|c|}{ Smokers ${ }^{\#}$} \\
\hline & Total & $D \leqslant 500^{\circ}$ & D $>500^{\circ}$ & $\mathbf{A C Q}<\mathbf{2}^{+}$ & $\Delta \mathrm{D} 0^{5}$ & Total & $D \leqslant 500^{\circ}$ & D $>500^{\circ}$ & $\mathbf{A C Q}<\mathbf{2}^{+}$ & $\Delta \mathrm{D} 0^{\S}$ \\
\hline Total events $n$ & 643 & 306 & 337 & 432 & 301 & 92 & 35 & 57 & 53 & 47 \\
\hline Positive cases $n$ & 257 & 116 & 141 & 112 & 108 & 40 & 14 & 26 & 18 & 15 \\
\hline Prevalence & 40 & 38 & 42 & 26 & 36 & 43 & 41 & 46 & 34 & 32 \\
\hline PPV & 61 & 58 & 60 & 41 & 47 & 62 & 75 & 59 & 56 & 57 \\
\hline NPV & 74 & 80 & 70 & 83 & 72 & 70 & 78 & 63 & 77 & 83 \\
\hline Accuracy & 68 & 70 & 66 & 66 & 65 & 66 & 77 & 61 & 70 & 72 \\
\hline p-value ${ }^{f}$ & $<0.001$ & $<0.001$ & $<0.001$ & $<0.001$ & 0.002 & $<0.001$ & $<0.001$ & 0.070 & $<0.001$ & $<0.001$ \\
\hline
\end{tabular}

Data are presented as \%, unless otherwise indicated. PPV: positive predictive value; NPV: negative predictive value. ${ }^{\#}$ : cut-off value of $-20 \%$; : inhaled corticosteroid (ICS) dose (D) in $\mu$ g equivalents of beclomethasone diproprionate day $^{-1}{ }^{+}{ }^{+}$: ACQ $<2$ tested the subgroup with an initial $A C Q$ score of $<2 ;{ }^{\varsigma}: \Delta D 0$ tested the subgroup without treatment modification between consecutive visits; ${ }^{f}$ : the $\mathrm{p}$-value was defined as the statistical significance of rejecting an area under the curve of 0.5 . A positive event was defined as an improvement in asthma control. A true-positive case was defined as an exhaled nitric oxide fraction (FeNO) change of less than or the same as a cut-off value (e.g. -25\%) associated with an improvement of asthma control between consecutive visits. When smoking patients were treated with a high ICS dose, FeNO lost its ability to assess a control improvement $(p=0.07)$. 


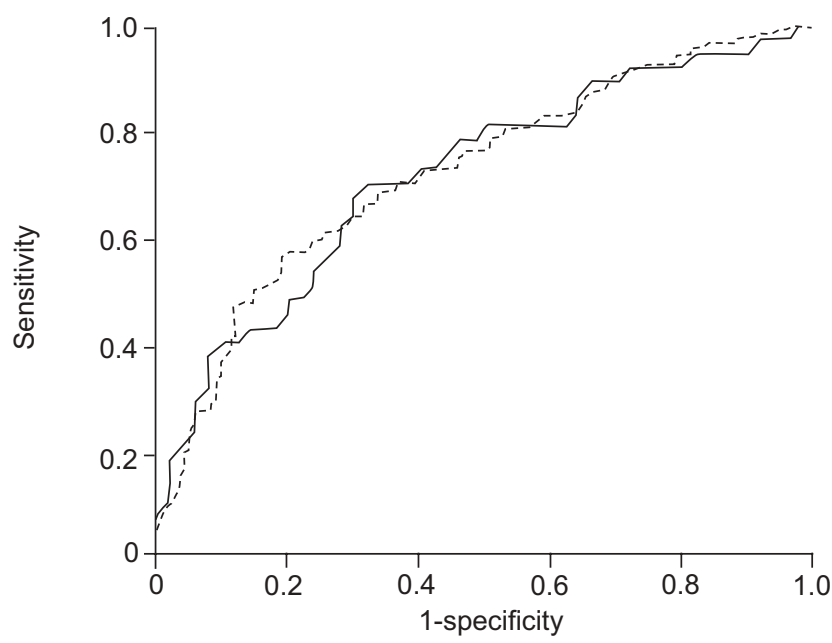

FIGURE 1. Receiver operated characteristic curve characterising the ability of exhaled nitric oxide fraction ( $F_{\mathrm{eNO}}$ ) to assess an improvement of asthma control defined as a significant Asthma Control Questionnaire (ACQ) score decrease $(\triangle A C Q$ score of $>0.5$ ) between two consecutive visits. FeNO exhibits similar operating characteristics in both populations. _- nonsmoking patients; ------: smoking patients.

modification. Overall, FeNO characteristics were found to be only mildly affected compared with the total group.

\section{DISCUSSION}

The present study confirms that, compared with nonsmokers, FeNO is reduced in smoking asthma patients. However, this reduction does not appear to suppress its ability to reflect asthma control in smoking patients, provided changes in FeNO values detected by repeated measurements are considered.

FeNO is a reliable marker of eosinophilic airway inflammation [26] and has the potential to be useful in the management of asthma [1-5]. However, tobacco smoking, which affects around $25 \%$ of the asthma population [17], leads to a decrease in FeNO $[6,8-15]$ and is considered to be a confounding factor. Therefore, it is generally assumed that FeNO should not be assessed in asthmatic patients who smoke.

At first glance, the present results seem to support this common paradigm. FeNO levels were in fact substantially reduced in smoking compared with nonsmoking asthma patients and to an extent that is similar to that found in previous studies [6, 14, 15]. Furthermore, whereas a single FeNO value was confirmed to be significantly related to asthma control in the nonsmoking population (i.e. FeNO level $>50 \mathrm{ppb}$ indicates uncontrolled asthma in most cases [18]), such a relationship could not be found in the smoking population.

However, in the present authors' previous study [18], which involved nonsmoking patients, it was shown that sequential FeNO assessments are more useful than isolated measurements in demonstrating asthma control. In the current study, this was also found to hold true for smoking asthma patients. Indeed, repeated FeNO measurements do appear helpful with regard to indicating change in asthma control over time in both populations. Thus, when asthma is uncontrolled in nonsmoking patients, an FeNO reduction of $>30 \%$ would predict that asthma is controlled in two out of three cases. The degree of change in FeNO that should be considered for smoking patients is different to that for nonsmoking patients: an FeNO reduction of $<20 \%$ would indicate that asthma remains uncontrolled in most cases. Conversely, when asthma is controlled, an FeNO increase of $<50 \%$ would indicate that asthma remains controlled in either population.

The aim of asthma treatment is to achieve full asthma control (i.e. an ACQ <0.75). In smoking patients, however, optimal control is usually more difficult to achieve [21, 27, 28]; this is most likely to be due to the reduction in anti-asthma drugs

TABLE 6 Assessment of asthma control worsening (change in Asthma Control Questionnaire $(\triangle \mathrm{ACQ})>+0.5)$

\begin{tabular}{|c|c|c|c|c|c|c|c|c|c|c|}
\hline & \multicolumn{5}{|c|}{ Nonsmokers ${ }^{\#}$} & \multicolumn{5}{|c|}{ Smokers ${ }^{\#}$} \\
\hline & Total & $D \leqslant 500^{\circ}$ & D $>500^{\circ}$ & $\mathbf{A C Q}<\mathbf{2}^{+}$ & $\Delta \mathrm{D} 0^{\S}$ & Total & $D \leqslant 500^{\circ}$ & $D>500^{\circ}$ & $\mathrm{ACQ}<\mathbf{2}^{+}$ & $\Delta \mathrm{D} 0^{\mathrm{s}}$ \\
\hline Total events $n$ & 643 & 306 & 337 & 432 & 301 & 92 & 35 & 57 & 53 & 47 \\
\hline Positive cases $\mathrm{n}$ & 161 & 64 & 97 & 130 & 99 & 26 & 11 & 15 & 17 & 14 \\
\hline Prevalence & 25 & 21 & 29 & 30 & 33 & 28 & 31 & 26 & 32 & 30 \\
\hline PPV & 37 & 43 & 44 & 47 & 34 & 52 & 78 & 43 & 71 & 47 \\
\hline NPV & 84 & 90 & 77 & 79 & 80 & 86 & 87 & 85 & 86 & 80 \\
\hline Accuracy & 70 & 74 & 68 & 67 & 65 & 74 & 86 & 70 & 81 & 68 \\
\hline p-value ${ }^{f}$ & $<0.001$ & $<0.001$ & $<0.001$ & $<0.001$ & $<0.001$ & $<0.001$ & $<0.001$ & 0.037 & $<0.001$ & 0.025 \\
\hline
\end{tabular}

Data are presented as \%, unless otherwise indicated. PPV: positive predictive value; NPV: negative predictive value. ${ }^{\#}$ : cut-off value of $+30 \%$; ": inhaled corticosteroid (ICS) dose (D) in $\mu \mathrm{g}$ equivalents of beclomethasone diproprionate $\cdot$ day $^{-1}{ }^{+}{ }^{+}$: ACQ $<2$ tested the subgroup with an initial ACQ score $<2 ;{ }^{\$}: \Delta D 0$ tested the subgroup without treatment modification between consecutive visits; ${ }^{f}$ : the $p$-value is the statistical significance of rejecting an area under the curve of 0.5 . A positive event is defined as a worsening of asthma control. A true-positive case was defined as an exhaled nitric oxide fraction ( $F$ eNO) change of more than or the same as the cut-off value associated with a worsening of asthma control between consecutive visits. When smoking patients were treated with a high ICS dose, FeNO ability to detect a worsening of control was somewhat reduced $(p=0.037)$. 
effectiveness that has recently been documented in this population [27, 28]. The present study confirms this: wellcontrolled asthma (ACQ score $<0.75$ ) was achieved in only $15 \%$ of smoking patients compared with $33 \%$ in nonsmokers $(\mathrm{p}<0.001$; data not shown). Treatment adjustments resulted in asthma that could no longer be considered poorly controlled in as many as $33 \%$ of smoking patients (data not shown). For this reason, the present authors felt that an ACQ cut-off score of 1.5 (which identifies poorly controlled asthma) was more appropriate for the present data analysis and, thus, selected it for the current study. As this level of control was achieved in only $33 \%$ of patients in the present study, the authors also considered the ability of FeNO to detect any significant improvement in asthma control [22]. In this respect, repeated FeNO assessments again appear to be helpful in both populations: in most cases, an FeNO reduction of $<20 \%$ indicates that no significant improvement in asthma control has occurred. Conversely, FeNO increases of $<30 \%$ are helpful to rule out mild deteriorations of asthma control. The results of the present study may be summarised as follows: if FeNO does not change as indicated, the level of asthma control is not modified. This seems to remain true regardless of whether the initial ACQ score was high or low, and whether or not the ICS dose was modified.

Interestingly, when patients were treated with high-to-medium ICS doses, FeNO no longer had the ability to reflect an improvement in asthma control for smoking patients, whereas for nonsmoking patients, its ability was only slightly reduced. A similar trend was observed with respect to asthma control deteriorations. These results confirm the overall reduction of the ability of FeNO to reflect asthma control in patients treated with high-to-medium ICS doses, as was documented by the present authors in their previous study [18]. In addition, it appears that confounding factors, such as high ICS doses [29, $30]$ and tobacco smoking $[6,8-15]$, which are known to reduce FeNO, would have a cumulative interfering effect that may eventually suppress the ability of FeNO to reflect asthma control. This suggests that the effect of these confounding factors might have to be taken into account when using FeNO to assess asthma control. This needs to be clarified by appropriately designed studies.

In conclusion, the present study is the first to indicate that cigarette smoking does not obviate the clinical value of measuring FeNO in asthma. Indeed, it is shown that even in smokers, sequential changes in FeNO have a relationship to asthma control. The results also suggest that factors, such as smoking and inhaled corticosteroid dose, act cumulatively to influence the ability of FeNO to be used to assess asthma control. Overall, the importance of sequential FeNO measurements in both smokers and nonsmokers is to distinguish whether or not ongoing changes or a sudden change in respiratory symptoms are/is due to changes in airway inflammation, possibly requiring a change in anti-inflammatory therapy. The present data provide evidence that enables the magnitude of changes in FeNO to be more accurately interpreted when addressing this important question.

\section{ACKNOWLEDGEMENTS}

The authors would like to thank E. Juniper (McMaster University, Hamilton, ON, Canada) for kindly allowing the authors to use the Asthma Control Questionnaire. The authors also thank M. Demosmaeker, M. Danschutter and J-P. Storms for technical assistance and D. Young (Young \& Associates Ltd, London, UK) for advice.

\section{REFERENCES}

1 Jones SL, Kittelson J, Cowan JO, et al. The predictive value of exhaled nitric oxide measurements in assessing changes in asthma control. Am J Respir Crit Care Med 2001; 164: 738-743.

2 Smith AD, Cowan JO, Brassett KP, et al. Use of exhaled nitric oxide measurements to guide treatment in chronic asthma. N Engl J Med 2005; 352: 2163-2173.

3 Shaw DE, Berry MA, Thomas M, et al. The use of exhaled nitric oxide to guide asthma management: a randomized controlled trial. Am J Respir Crit Care Med 2007; 176: 231-237.

4 Turner S. Exhaled nitric oxide in the diagnosis and management of asthma. Curr Opin Allergy Clin Immunol 2008; 8: 70-76.

5 Szefler SJ, Mitchell H, Sorkness CA, et al. Management of asthma based on exhaled nitric oxide in addition to guideline-based treatment for inner-city adolescents and young adults: a randomised controlled trial. Lancet 2008; 372: 1065-1072.

6 Olin AC, Rosengren A, Thelle DS, et al. Height, age, and atopy are associated with fraction of exhaled nitric oxide in a large adult general population sample. Chest 2006; 130: 1319-1325.

7 Taylor DR, Pijnenburg MW, Smith AD, et al. Exhaled nitric oxide measurements: clinical application and interpretation. Thorax 2006; 61: 817-827.

8 Persson MG, Zetterstrom O, Agrenius V, et al. Singlebreath nitric oxide measurements in asthmatic patients and smokers. Lancet 1994; 343: 146-147.

9 Kharitonov SA, Robbins RA, Yates D, et al. Acute and chronic effects of cigarette smoking on exhaled nitric oxide. Am J Respir Crit Care Med 1995; 152: 609-612.

10 Verleden GM, Dupont LJ, Verpeut AC, et al. The effect of cigarette smoking on exhaled nitric oxide in mild steroidnaive asthmatics. Chest 1999; 116: 59-64.

11 Hogman M, Holmkvist $T$, Walinder R, et al. Increased nitric oxide elimination from the airways after smoking cessation. Clin Sci (Lond) 2002; 103: 15-19.

12 Horvath I, Donnelly LE, Kiss A, et al. Exhaled nitric oxide and hydrogen peroxide concentrations in asthmatic smokers. Respiration 2004; 71: 463-468.

13 McSharry CP, McKay IC, Chaudhuri R, et al. Short and long-term effects of cigarette smoking independently influence exhaled nitric oxide concentration in asthma. $J$ Allergy Clin Immunol 2005; 116: 88-93.

14 Travers J, Marsh S, Aldington S, et al. Reference ranges for exhaled nitric oxide derived from a random community survey of adults. Am J Respir Crit Care Med 2007; 176: 238-242.

15 Dressel H, de la Motte D, Reichert J, et al. Exhaled nitric oxide: independent effects of atopy, smoking, respiratory tract infection, gender and height. Respir Med 2008; 102: 962-969. 
16 Gaston B, Drazen JM, Loscalzo J, et al. The biology of nitrogen oxides in the airways. Am J Respir Crit Care Med 1994; 149: 538-551.

17 Thomson NC, Chaudhuri R, Livingston E. Asthma and cigarette smoking. Eur Respir J 2004; 24: 822-833.

18 Michils A, Baldassarre S, Van Muylem A. Exhaled nitric oxide and asthma control: a longitudinal study in unselected patients. Eur Respir J 2008; 31: 539-546.

19 Juniper EF, O’Byrne PM, Guyatt GH, et al. Development and validation of a questionnaire to measure asthma control. Eur Respir J 1999; 14: 902-907.

20 Global initiative for Asthma. Global strategy for asthma management and prevention: NHLBI/WHO Workshop Report. NHLBI Publication 02-3659. Washington, Government Printing Office, 2002.

21 Siroux V, Pin I, Oryszczyn MP, et al. Relationships of active smoking to asthma and asthma severity in the EGEA study. Epidemiological study on the Genetics and Environment of Asthma. Eur Respir J 2000; 15: 470-477.

22 Juniper EF, Bousquet J, Abetz L, et al. Identifying "wellcontrolled" and "not well-controlled" asthma using the Asthma Control Questionnaire. Respir Med 2006; 100: 616-621.

23 Juniper EF, Svensson K, Mork AC, et al. Measurement properties and interpretation of three shortened versions of the asthma control questionnaire. Respir Med 2005; 99: 553-558.
24 American Thoracic Society/European Respiratory Society. ATS/ERS recommendations for standardized procedures for the online and offline measurement of exhaled lower respiratory nitric oxide and nasal nitric oxide, 2005. Am J Respir Crit Care Med 2005; 171 912-930.

25 Youden WJ. Index for rating diagnostic tests. Cancer 1950; 3: $32-35$

26 Jatakanon A, Lim S, Kharitonov SA, et al. Correlation between exhaled nitric oxide, sputum eosinophils, and methacholine responsiveness in patients with mild asthma. Thorax 1998; 53: 91-95.

27 Chalmers GW, Macleod KJ, Little SA, et al. Influence of cigarette smoking on inhaled corticosteroid treatment in mild asthma. Thorax 2002; 57: 226-230.

28 Lazarus SC, Chinchilli VM, Rollings NJ, et al. Smoking affects response to inhaled corticosteroids or leukotriene receptor antagonists in asthma. Am J Respir Crit Care Med 2007; 175: 783-790.

29 Jatakanon A, Kharitonov S, Lim S, et al. Effect of differing doses of inhaled budesonide on markers of airway inflammation in patients with mild asthma. Thorax 1999; 54: 108-114.

30 Jones SL, Herbison P, Cowan JO, et al. Exhaled NO and assessment of anti-inflammatory effects of inhaled steroid: dose-response relationship. Eur Respir J 2002; 20: 601-608. 